\title{
Integrable Singularities and \\ Weakly Sequentially Continuous Maps
}

\author{
R. P. Agarwal and D. O'Regan
}

\begin{abstract}
New existence results for singular second order boundary value problems, where the singularity is integrable, are presented using fixed point theory for weakly sequentially continuous maps.

Keywords: Integrable singularities, existence of solutions, fixed points, weak sequential continuity
\end{abstract}

AMS subject classification: 47A10, 34B16

\section{Introduction}

In this paper we discuss the singular boundary value problem

$$
\left.\begin{array}{l}
y^{\prime \prime}+f(t, y)=0 \quad \text { a.e. on }[0,1] \\
y(0)=y(1)=0
\end{array}\right\}
$$

where our non-linearity $f$ may be singular in the independent variable $y$ and may also be singular at $y=0$. Problems of form (1.1) have received a lot of attention in the literature, see $[1,3,5,8,10]$ and the references therein. All of these papers make use of fixed point theory for continuous, compact maps. In his 1991 book, Corduneanu [7: pp. 192] (based on work of Gaponenko [9]) shows how fixed point theory for weakly sequentially continuous maps can be used to discuss non-singular problems of form (1.1). These ideas were extended by Bonanno [6] to singular problem (1.1) where the boundary condition $y(0)=$ $y(1)=0$ is replaced by $y(0)=0, y(1)=a>0$.

R. P.Agarwal: Florida Inst. Techn., Dept. Math., Melbourne, Florida 32901, USA D. O'Regan: Nat. Univ. of Ireland, Dept. Math., Galway, Ireland agarwal@fit.edu and donal.oregan@nuigalway.ie

ISSN 0232-2064 / \$2.50 C Heldermann Verlag Berlin 
In the present paper we use fixed point theory for weakly sequentially continuous maps to present existence results for problem (1.1) where the singularity is integrable. We also indicate how these results could be obtained using fixed point theory for continuous, compact maps. However, in our opinion, the weakly sequentially continuous approach is easier and quicker since one does not need to check the compactness of the map. The results of this paper improve and extend the results in [6]. Moreover, it is easy to see that we could consider Sturm-Liouville boundary data also in (1.1); however, since the arguments are essentially the same we will restrict our discussion to Dirichlet data. In fact, homogeneous Dirichlet data are the most difficult boundary data to discuss in the singular case. In Section 2 we also discuss the nonsingular problem (1.1) and we present two results based on fixed point theory for weakly sequentially continuous maps. We also remark here that all the results in this paper easily extend to higher order boundary value problems (see Theorem 2.6). Finally, we present the fixed point theorems which will be needed in Section 2.

The first result is due to Arino, Gautier and Penot [4].

Theorem 1.1. Let $E$ be a metrizable locally convex linear topological space and let $Q$ be a weakly compact, convex subset of $E$. Then any weakly sequentially continuous map $F: Q \rightarrow Q$ has a fixed point.

Our next result is a Furi-Pera theorem for weakly sequentially continuous maps. This result can be found in [11]; we note that one of the conditions is stated incorrectly and the proof there has to be adjusted slightly (see [2]).

Theorem 1.2. Let $E$ be a separable and reflexive Banach space, and let $C$ and $Q$ be closed bounded convex subsets of $E$ with $Q \subseteq C$ and $0 \in Q$. Suppose $F: Q \rightarrow C$ is a weakly sequentially continuous map and assume the following condition is satisfied:

$$
\left\{\begin{array}{l}
\text { If }\left\{\left(x_{j}, \lambda_{j}\right)\right\}_{1}^{\infty} \text { is a sequence in } Q \times[0,1] \text { with } \\
x_{j} \rightarrow x, \lambda_{j} \rightarrow \lambda \text { and } x=\lambda F(x) \text { for } 0 \leq \lambda<1 \\
\text { then there exists } j_{0} \in \mathbb{N} \text { with } \lambda_{j_{0}} F\left(x_{j_{0}}\right) \in Q
\end{array}\right\}
$$

Then $F$ has a fixed point in $Q$.

\section{Existence theory}

Our first result concerns problem (1.1) when $f$ may be singular in the dependent variable. We note that $f$ may be singular also in the independent variable at some set $\Omega \subseteq[0,1]$ with measure zero. 
Theorem 2.1. Suppose the following conditions are satisfied:

$$
\begin{gathered}
\left\{\begin{array}{l}
f:[0,1] \times(0, \infty) \rightarrow \mathbb{R} \text { with } \\
t \mapsto f(t, y) \text { measurable } \forall y \in(0, \infty) \\
y \mapsto f(t, y) \text { continuous for a.e. } t \in(0,1)
\end{array}\right\} \\
\left\{\begin{array}{l}
\forall r>0 \exists \psi_{r}:[0,1] \rightarrow \mathbb{R} \text { with } \\
\psi_{r}>0 \text { a.e. on }[0,1], \psi_{r} \in L^{1}[0,1] \\
f(t, y) \geq \psi_{r}(t) \text { a.e. on }[0,1] \forall y \in(0, r]
\end{array}\right\} \\
\left\{\begin{array}{l}
\forall r>0 \exists h_{r}:[0,1] \rightarrow \mathbb{R} \text { with } \\
h_{r} \geq 0 \text { a.e. on }[0,1], h_{r} \in L^{1}[0,1] \\
f(t, y) \leq h_{r}(t) \text { for a.e. } t \in[0,1] \text { and } y \in\left[\int_{0}^{1} G(t, s) \psi_{r}(s) d s, r\right]
\end{array}\right\}
\end{gathered}
$$

and

$$
\exists M>0 \text { with } M \geq \int_{0}^{1} G(s, s) h_{M}(s) d s
$$

where

$$
G(t, s)= \begin{cases}(1-t) s & \text { if } 0 \leq s \leq t \leq 1 \\ (1-s) t & \text { if } 0 \leq t \leq s \leq 1\end{cases}
$$

Then problem (1.1) has a solution $y \in W^{2,1}[0,1]$ with $y(t)>0$ for $t \in(0,1)$.

Remark 2.1. In Theorem 2.1 it is possible to replace condition (2.3) with the following one:

$$
\left\{\begin{array}{l}
\text { For any } r>0, \text { assume } h_{r} \in L^{1}[0,1] \text { where } \\
h_{r}(t)=\sup \left\{f(t, y): y \in\left[\int_{0}^{1} G(t, s) \psi_{r}(s) d s, r\right]\right\}
\end{array}\right\} .
$$

Remark 2.2. In Theorem 2.1 notice that the solution $y$ to problem (1.1) satisfies $y(t) \geq \int_{0}^{1} G(t, s) \psi_{M}(s) d s$ for $t \in[0,1]$ (see the proof) and, moreover, it is easy to see (see the argument in Theorem 2.2) that there exists a constant $k_{M}>0$ with $y(t) \geq k_{M} t(1-t)$ for $t \in[0,1]$.

Proof of Theorem 2.1. Let the constant $M$ be chosen as in condition (2.4), and choose the functions $\psi_{M}$ and $h_{M}$ as in (2.2) and (2.3), respectively. Let

$$
Q=\left\{u \in L^{1}[0,1]: \psi_{M}(t) \leq u(t) \leq h_{M}(t) \text { for a.e. } t \in[0,1]\right\} .
$$

Clearly, $Q$ is convex and closed, so weakly closed. Indeed, to see closedness let $v_{n} \in Q$ with $v_{n} \rightarrow v$ in $L^{1}[0,1]$. Since there exists a subsequence $S$ of $\mathbb{N}$ with $v_{n}(t) \rightarrow v(t)$ a.e. on $[0,1]$ as $n \rightarrow \infty$ in $S$ we have immediately that $v \in Q$. In fact, $Q$ is weakly compact by the Dunford-Pettis theorem. 
We will apply Theorem 1.1. With this in mind define the operator $\Phi$ : $L^{1}[0,1] \rightarrow C[0,1]$ by

$$
(\Phi u)(t)=\int_{0}^{1} G(t, s) u(s) d s
$$

It is easy to see that solving problem (1.1) is equivalent to finding a solution $u \in L^{1}[0,1]$ to the equation

$$
u=f(t, \Phi(u))
$$

For this define the operator $F: L^{1}[0,1] \rightarrow L^{1}[0,1]$ by $(F u)(t)=f(t, \Phi(u)(t))$. So solving (2.6) is equivalent to finding a fixed point of $F$.

First we show that $F: Q \rightarrow Q$. To see this let $u \in Q$. So $\psi_{M}(t) \leq u(t) \leq$ $h_{M}(t)$ for a.e. $t \in[0,1]$. Notice from $(2.4)$ that

$$
(\Phi u)(t) \geq \int_{0}^{1} G(t, s) \psi_{M}(s) d s
$$

and

$$
(\Phi u)(t) \leq \int_{0}^{1} G(t, s) h_{M}(s) d s \leq \int_{0}^{1} G(s, s) h_{M}(s) d s \leq M .
$$

So as a result, (2.2) and (2.3) imply

$$
\psi_{M}(t) \leq f(t, \Phi(u)(t)) \leq h_{M}(t) \quad \text { for a.e. } t \in[0,1]
$$

Thus $F u \in Q$, so $F: Q \rightarrow Q$.

It remains to show that $F: Q \rightarrow Q$ is weakly sequentially continuous. Let $\left\{y_{n}\right\}$ be a sequence in $Q$ with $y_{n} \rightarrow y$ in $L^{1}[0,1]$. Notice for fixed $t \in[0,1]$ that (note that $G(t, \cdot) \in L^{\infty}[0,1]$ )

$$
\left|\left(\Phi y_{n}\right)(t)-(\Phi y)(t)\right|=\left|\int_{0}^{1} G(t, s)\left[y_{n}(t)-y(s)\right] d s\right| \rightarrow 0 \quad \text { as } n \rightarrow \infty
$$

so $\lim _{n \rightarrow \infty}\left(\Phi y_{n}\right)(t)=(\Phi y)(t)$. From $(2.1)$, the sequence $\left\{F y_{n}\right\}$ converges to $F y$ a.e. on $[0,1]$. Also, $F y_{n} \in Q$. Thus $\left|\left(F y_{n}\right)(t)\right| \leq h_{M}(t)$ for a.e. $t \in[0,1]$. The Lebesgue dominated convergence theorem implies

$$
\lim _{n \rightarrow \infty} F y_{n}=F y \quad \text { in } L^{1}[0,1]
$$

so in particular $F y_{n} \rightarrow F y$ in $L^{1}[0,1]$. Now Theorem 1.1 guarantees that equation (2.6) has a solution $u \in Q$. As a result we notice that $y=\Phi u$ satisfies (1.1) with $y(t) \geq \int_{0}^{1} G(t, s) \psi_{M}(s) d s>0$ for $t \in(0,1)$. Note also that $y \in W^{2,1}[0,1]$ 
Remark 2.3. Notice that one could also prove Theorem 2.1 using Schauder's fixed point theorem. To see this take $Q$ to be the set

$$
\left\{u \in C[0,1]: \int_{0}^{1} G(t, s) \psi_{M}(s) d s \leq u(t) \leq \int_{0}^{1} G(t, s) h_{M}(s) d s \quad(t \in[0,1])\right\}
$$

and let $F: C[0,1] \rightarrow C[0,1]$ be defined by $(F y)(t)=\int_{0}^{1} G(t, s) f(s, y(s)) d s$.

Our next result is a more "applicable" version of Theorem 2.1.

Theorem 2.2. Suppose conditions (2.1) - (2.2) hold and in addition suppose the following conditions are satisfied:

$$
\begin{gathered}
\left\{\begin{array}{l}
f(t, y) \leq q(t)[g(y)+\tau(y)] \text { on }[0,1] \times(0, \infty) \text { with } \\
g>0 \text { continuous and non-increasing on }(0, \infty) \\
\tau \geq 0 \text { continuous and non-decreasing on }(0, \infty) \\
q:[0,1] \rightarrow \mathbb{R} \text { with } q>0 \text { a.e. on }[0,1]
\end{array}\right\} \\
\int_{0}^{1} q(s) g\left(a_{0} s(1-s)\right) d s<\infty \quad \text { for any } a_{0}>0
\end{gathered}
$$

and there exists a constant $M>0$ with

$$
\int_{0}^{1} G(s, s) q(s)\left[\tau(M)+g\left(\int_{0}^{1} G(s, x) \psi_{M}(x) d x\right)\right] d s \leq M
$$

where $G$ is as in Theorem 2.1. Then problem (1.1) has a solution $y \in W^{2,1}[0,1]$ with $y(t)>0$ for $t \in(0,1)$.

Proof. The result follows from Theorem 2.1 once we show that conditions (2.3) - (2.4) hold. Notice that for $y \in\left[\int_{0}^{1} G(t, s) \psi_{r}(s) d s, r\right]$ and a.e. $t \in[0,1]$ condition (2.9) yields

$$
f(t, y) \leq q(t)\left[\tau(r)+g\left(\int_{0}^{1} G(t, s) \psi_{r}(s) d s\right)\right] .
$$

If we denote the right-hand side by $h_{r}(t)$, then condition (2.3) will be immediate if we show that $h_{r} \in L^{1}[0,1]$. To see this notice that

$$
\int_{0}^{1} G(t, s) \psi_{r}(s) d s=t(1-t) \Theta_{r}(t)
$$

where $\Theta_{r}(t)=\frac{1}{1-t} \int_{t}^{1}(1-s) \psi_{r}(s) d s+\frac{1}{t} \int_{0}^{t} s \psi_{r}(s) d s$. Now since

$$
\begin{aligned}
\left|\frac{1}{t} \int_{0}^{t} s \psi_{r}(s) d s\right| & \leq \int_{0}^{t} \psi_{r}(s) d s \rightarrow 0 & \text { as } t \rightarrow 0^{+} \\
\left|\frac{1}{1-t} \int_{t}^{1}(1-s) \psi_{r}(s) d s\right| & \leq \int_{t}^{1} \psi_{r}(s) d s \rightarrow 0 & \text { as } t \rightarrow 1^{-},
\end{aligned}
$$


$\Theta_{r}$ extends to a continuous function on $[0,1]$. Thus there exists $k_{r}>0$ with $\Theta_{r}(t) \geq k_{r}>0$ for $t \in[0,1]$. As a result

$$
\int_{0}^{1} G(t, s) \psi_{r}(s) d s \geq k_{r} t(1-t) \quad \text { for } t \in[0,1]
$$

Thus $h_{r}(t) \leq q(t)\left[\tau(r)+g\left(k_{r} t(1-t)\right)\right]$ and $h_{r} \in L^{1}[0,1]$ from condition $(2.10)$. Notice that condition (2.4) is immediate since

$$
\begin{aligned}
\int_{0}^{1} G(s, s) h_{M}(s) d s \\
\quad=\int_{0}^{1} G(s, s) q(s)\left[\tau(M)+g\left(\int_{0}^{1} G(s, x) \psi_{M}(x) d x\right)\right] d s .
\end{aligned}
$$

Thus the theorem is proved

To show how Theorem 2.2 can be applied in practice consider the problem

$$
\left.\begin{array}{rl}
y^{\prime \prime}+q(t)[g(y)+\tau(y)] & =0 \text { a.e. on }[0,1] \\
y(0)=y(1) & =0
\end{array}\right\} .
$$

Theorem 2.3. Assume the following conditions are satisfied:

$$
\begin{gathered}
\left\{\begin{array}{l}
g>0 \text { is continuous and non-increasing on }(0, \infty) \\
\tau \geq 0 \text { is continuous and non-decreasing on }(0, \infty) \\
q:[0,1] \rightarrow \mathbb{R} \text { is measurable with } q>0 \text { a.e. on }[0,1]
\end{array}\right\} \\
\int_{0}^{1} q(s) g\left(a_{0} s(1-s)\right) d s<\infty \text { for any } a_{0}>0
\end{gathered}
$$

and there exists a constant $M>0$ with

$$
\int_{0}^{1} G(s, s) q(s)\left[\tau(M)+g\left(g(M) \int_{0}^{1} G(s, x) q(x) d x\right)\right] d s \leq M
$$

where $G$ is as in Theorem 2.1. Then problem (2.12) has a solution $y \in$ $W^{2,1}[0,1]$ with $y(t)>0$ for $t \in(0,1)$.

Proof. The result follows from Theorem 2.2 once we notice that we can take $\psi_{r}(t)=q(t) g(r)$ 
Remark 2.4. If $g(y)=y^{-\alpha}$ with $\alpha>0$ and for $x \geq 0$ we have $\tau(x) \leq$ $A x^{p}+B$ with $A, B, p \geq 0$, then condition (2.15) reduces to

$$
\int_{0}^{1} G(s, s) q(s)\left[A M^{p}+B+M^{\alpha^{2}}\left(\int_{0}^{1} G(s, x) q(x) d x\right)^{-\alpha}\right] d s \leq M .
$$

Of course, if $\alpha<1$ and $p<1$, then this inequality is satisfied for $M$ large.

It is also possible to improve Theorem 2.1 if $f$ is non-singular in the dependent variable, i.e. $f:[0,1] \times[0, \infty) \rightarrow \mathbb{R}$.

Theorem 2.4. Suppose the following conditions are satisfied:

$$
\begin{aligned}
& \left\{\begin{array}{l}
f:[0,1] \times[0, \infty) \rightarrow \mathbb{R} \text { with } \\
t \mapsto f(t, y) \text { measurable for every } y \in[0, \infty) \\
y \mapsto f(t, y) \text { continuous for a.e. } t \in(0,1)
\end{array}\right\} \\
& \left\{\begin{array}{l}
\forall r>0 \exists h_{r}:[0,1] \rightarrow \mathbb{R} \text { with } \\
h_{r} \geq 0 \text { a.e. on }[0,1], h_{r} \in L^{1}[0,1] \\
0 \leq f(t, y) \leq h_{r}(t) \text { a.e. on }[0,1] \forall y \in[0, r]
\end{array}\right\}
\end{aligned}
$$

and

$$
\exists M>0 \text { with } M \geq \int_{0}^{1} G(s, s) h_{M}(s) d s
$$

where $G$ is as in Theorem 2.1. Then problem (1.1) has a solution $y \in W^{2,1}[0,1]$ with $y(t) \geq 0$ for $t \in[0,1]$.

Proof. Let the constant $M$ be chosen as in (2.18), let

$$
Q=\left\{u \in L^{1}[0,1]: 0 \leq u(t) \leq h_{M}(t) \text { for a.e. } t \in[0,1]\right\}
$$

and let $F$ be as in Theorem 2.1. It is easy to check (as in Theorem 2.1) that $F: Q \rightarrow Q$ is weakly sequentially continuous

If we are not particularly interested in non-negative solutions, it is easy to modify Theorem 2.4 and to consider maps $f:[0,1] \times \mathbb{R} \rightarrow \mathbb{R}$. Our next result replaces (2.18) with a less restrictive condition, and for completeness we discuss the existence of a solution which is not necessarily non-negative.

Theorem 2.5. Let $1<p<\infty$ and suppose the following conditions are satisfied:

$$
\left\{\begin{array}{l}
f:[0,1] \times \mathbb{R} \rightarrow \mathbb{R} \text { with } \\
t \mapsto f(t, y) \text { measurable } \forall y \in \mathbb{R} \\
y \mapsto f(t, y) \text { continuous for a.e. } t \in(0,1)
\end{array}\right\}
$$




$$
\begin{aligned}
& \left\{\begin{array}{l}
\forall r>0 \exists h_{r}:[0,1] \rightarrow \mathbb{R} \text { with } \\
h_{r} \geq 0 \text { a.e. on }[0,1], h_{r} \in L^{p}[0,1] \\
|f(t, y)| \leq h_{r}(t) \text { a.e. on }[0,1] \forall|y| \leq r
\end{array}\right\} \\
& \left\{\begin{array}{l}
\exists M>0 \text { with }\left\|y^{\prime \prime}\right\|_{L^{p}[0,1]} \leq M \forall \text { solutions } y \in W^{2,1}[0,1] \text { to } \\
y^{\prime \prime}+\lambda f(t, y)=0 \text { a.e. on }[0,1] \text { and } y(0)=y(1)=0 \forall \lambda \in(0,1)
\end{array}\right\} .
\end{aligned}
$$

Then problem (1.1) has a solution $y \in W^{2,1}[0,1]$.

Proof. Let the constant $M$ be as in condition (2.21) and

$$
Q=\left\{u \in L^{p}[0,1]:\|u\|_{L^{p}[0,1]} \leq M+1\right\}
$$

Also, let $M_{1}=[M+1]\left(\int_{0}^{1}\left[G(s, s]^{q} d s\right)^{1 / q}\right.$ where $G$ is as in Theorem 2.1 and $\frac{1}{p}+\frac{1}{q}=1$. Now let

$$
C=\left\{u \in L^{p}[0,1]:|u(t)| \leq h_{M_{1}}(t) \text { a.e. on }[0,1]\right\}
$$

Finally, let $F$ be as in Theorem 2.1. First we show that $F: Q \rightarrow C$. To see this let $y \in Q$ so that $\|y\|_{L^{p}[0,1]} \leq M+1$. Notice that for $t \in[0,1]$

$$
|\Phi(y)(t)|=\left|\int_{0}^{1} G(t, s) y(s) d s\right| \leq \int_{0}^{1} G(s, s)|y(s)| d s
$$

so that

$$
\begin{aligned}
|\Phi(y)|_{0} & =\sup _{t \in[0,1]}|\Phi(y)(t)| \\
& \leq\|y\|_{L^{p}[0,1]}\left(\int_{0}^{1}[G(s, s)]^{q} d s\right)^{\frac{1}{q}} \\
& \leq[M+1]\left(\int_{0}^{1}[G(s, s)]^{q} d s\right)^{\frac{1}{q}} \\
& =M_{1} .
\end{aligned}
$$

This together with condition (2.20) yields $|F y(t)|=|f(t, \Phi(y)(t))| \leq h_{M_{1}}(t)$ a.e. on $[0,1]$, so $F y \in C$. It is easy to check (as in Theorem 2.1) that $F: Q \rightarrow C$ is weakly sequentially continuous.

The result follows from Theorem 1.2 once we check condition (1.2). For this take a sequence $\left\{\left(x_{j}, \lambda_{j}\right)\right\}_{j=1}^{\infty}$ in $Q \times[0,1]$ with $\lambda_{j} \rightarrow \lambda$ and $x_{j} \rightarrow x$, with $x=\lambda F x$ for $0 \leq \lambda<1$. The argument used to prove (2.8) implies $\lim _{j \rightarrow \infty} F x_{j}=F x$ in $L^{p}[0,1]$. Now given $\varepsilon>0$ (say $\varepsilon<\frac{1}{3}$ ), there exists 
$j_{0} \in \mathbb{N}$ with $\left\|F x_{j}\right\|_{L^{p}[0,1]} \leq\|F x\|_{L^{p}[0,1]}+\varepsilon$ for $j \geq j_{0}$. This together with $x=\lambda F x$ and $\|x\|_{L^{p}[0,1]} \leq M$ (see condition (2.21)) yields

$$
\begin{aligned}
\left\|\lambda_{j} F x_{j}\right\|_{L^{p}[0,1]} & \leq\left|\lambda_{j}-\lambda\right|\|F x\|_{L^{p}[0,1]}+\|x\|_{L^{p}[0,1]}+\varepsilon \\
& \leq\left|\lambda_{j}-\lambda\right|\left\|h_{M_{1}}\right\|_{L^{p}[0,1]}+M+\varepsilon
\end{aligned}
$$

for $j \geq j_{0}$. Now since $\lambda_{j} \rightarrow \lambda$, there exists $j_{0} \leq j_{1} \in \mathbb{N}$ with $\left\|\lambda_{j} F x_{j}\right\|_{L^{p}[0,1]} \leq$ $M+1$ for $j \geq j_{1}$. As a result, $\lambda_{j} F x_{j} \in Q$ for $j \in \mathbb{N}$ sufficiently large, so condition (1.2) holds. Thus we may apply Theorem 1.2 to get the conclusion

All the results in this paper easily extend to higher order boundary value problems. To see this we consider the Fredholm integral equation (which includes all higher order boundary value problems)

$$
y(t)=\int_{0}^{1} k(t, s) f(s, y(s)) d s \quad \text { for } t \in[0,1] .
$$

Essentially the same reasoning as in Theorem 2.1 (see Remark 2.3) establishes the following result.

Theorem 2.6. Let $1 \leq p \leq \infty$ and let $q$ be the conjugate to $p$. Suppose the following conditions are satisfied:

$$
\begin{aligned}
& \left\{\begin{array}{l}
\text { For all } t \in[0,1], k_{t}(s)=k(t, s) \geq 0 \text { for a.e. } s \in[0,1] \\
\text { and for a.e. } t \in[0,1], k_{t}(s)>0 \text { for a.e. } s \in[0,1]
\end{array}\right\} \\
& \left\{\begin{array}{l}
k_{t} \in L^{p}[0,1] \text { for each } t \in[0,1] \text { and } \\
\text { the map } t \mapsto k_{t} \text { is continuous from }[0,1] \text { to } L^{p}[0,1]
\end{array}\right\} .
\end{aligned}
$$

Also, assume condition (2.1) holds and suppose the following conditions are satisfied:

$$
\begin{gathered}
\left\{\begin{array}{l}
\forall r>0 \exists \psi_{r}:[0,1] \rightarrow \mathbb{R} \text { with } \\
\psi_{r}>0 \text { a.e. on }[0,1], \psi_{r} \in L^{q}[0,1] \\
f(t, y) \geq \psi_{r}(t) \text { a.e. on }[0,1] \forall y \in(0, r]
\end{array}\right\} \\
\left\{\begin{array}{l}
\forall r>0 \exists h_{r}:[0,1] \rightarrow \mathbb{R} \text { with } \\
h_{r} \geq 0 \text { a.e. on }[0,1], h_{r} \in L^{q}[0,1] \\
f(t, y) \leq h_{r}(t) \text { for a.e. } t \in[0,1] \text { and } y \in\left[\int_{0}^{1} k(t, s) \psi_{r}(s) d s, r\right]
\end{array}\right\}
\end{gathered}
$$

and

$$
\exists M>0 \text { with } M \geq \sup _{t \in[0,1]} \int_{0}^{1} k(t, s) h_{M}(s) d s .
$$

Then equation (2.22) has a solution $y \in C[0,1]$ with $y(t)>0$ for a.e. $t \in[0,1]$. 


\section{References}

[1] Agarwal, R. P. and D. O'Regan: Existence theory for single and multiple solutions to singular positone boundary value problems. J. Diff. Equ. 175 (2001), $393-414$.

[2] Agarwal, R. P. and D. O'Regan: Fixed point theory for weakly sequentially upper semicontinuous maps with applications to differential inclusions. Nonlin. Oscillations (to appear).

[3] Agarwal, R. P., O'Regan, D. and P. J. Y. Wong: Positive Solutions of Differential, Difference and Integral Equations. Dordrecht: Kluwer Acad. Publ. 1999.

[4] Arino, O., Gautier, S. and J. P. Penot: A fixed point theorem for sequentially continuous mappings with applications to ordinary differential equations. Funkc. Ekvac. 27 (1984), 273 - 279.

[5] Bobisud, L. E., Calvert, J. E. and W. D. Royalty: Some existence results for singular boundary value problems. Diff. \& Int. Equ. 6 (1993), 553 - 571.

[6] Bonanno, G.: An existence theorem for positive solutions to a singular nonlinear boundary value problem. Comment. Math. Univ. Carolinae 36 (1995), 609 614.

[7] Corduneanu, C.: Integral Equations and Applications. Cambridge Univ. Press 1991.

[8] Eloe, P. W. and J. Henderson: Singular nonlinear boundary value problems for higher order ordinary differential equations. Nonlin. Anal. 17 (1991), 1 - 10.

[9] Gaponenko, Y. L.: A condition on the solvability of quasilinear boundary value problems. Diff. Equ. (Transl.) 19 (1984), 1234 - 1238.

[10] Luning, C. D. and W. L. Perry: Positive solutions of negative exponent generalized Emden-Fowler boundary value problems. SIAM J. Math. Anal. 12 (1981), $874-879$.

[11] O'Regan, O.: A continuation method for weakly condensing operators. Z. Anal. Anw. 15 (1996), $565-578$.

Received 25.06.2002; in revised form 01.11.2002 